12,18

\title{
Особенности распространения плазмонов в графеновом бислое в условиях поперечного электрического поля
}

\author{
(C) Е.И. Кухарь ${ }^{1,2}$, С.В. Крючков ${ }^{1,2}$ \\ ${ }^{1}$ Волгоградский государственный социально-педагогический университет, \\ Волгоград, Россия \\ ${ }^{2}$ Волгоградский государственный технический университет, \\ Волгоград, Россия \\ E-mail: eikuhar@yandex.ru
}

Поступила в Редакцию 22 мая 2019 г.

В окончательной редакции 23 мая 2019 г.

Принята к публикации 3 сентября 2019 г.

Исследовано дисперсионное соотношение для плазменных волн в графеновом бислое. В приближении случайных фаз изучено влияние разности потенциалов между графеновыми слоями на кривизну дисперсионной линии для плазмонов. Показана возможность управления энергией и групповой скоростью плазмонов за счет изменения указанной разности потенциалов. Продемонстрирован немонотонный характер зависимости энергии плазмонов от напряжения между графеновыми слоями. Проанализирована температурная зависимость закона дисперсии плазмонов.

Ключевые слова: графеновый бислой, плазменные волны, плазмоны.

DOI: 10.21883/FTT.2020.01.48753.487

\section{Введение}

Современные достижения как в области физики твердого тела [1], так и в области наноэлектроники [2] дают возможность для управления электронными свойствами различных низкоразмерных структур за счет изменения внешних электромагнитных (ЭМ) полей. Одной из основных целей электронной инженерии является создание электронных устройств с перестраиваемыми характеристиками. В настоящее время особое внимание исследователей направлено на так называемые двумерные (2D) дираковские (или графеноподобные) материалы. К ним относятся, например, графеновые структуры, силицен, германен, фосфорен и т.д. Тем не менее, бесщелевая зонная структура некоторых таких материалов (в том числе свободного графенового монослоя) делают их неприменимыми в области полупроводниковой наноэлектроники.

По сравнению с графеном для носителей заряда которого характерна линейная дисперсионная зависимость в окрестности дираковских точек $(K)$, носители заряда в графеновом бислое (биграфене) обладают ненулевой эффективной массой. Кроме того, имеется возможность возникновения энергетической щели между валентной зоной и зоной проводимости, если биграфен помещен в электрическое поле, силовые линии которого перпендикулярны его плоскости [3]. В такой ситуации электронные свойства биграфена определяются величиной разности потенциалов между графеновыми слоями. Эту разность потенциалов будем называть смещающим напряжением (CH). Последнее обстоятельство делает биграфен весьма перспективным материалом для нано- электроники. В $[3,4]$ показано, что минимум энергии электронов в биграфене с $\mathrm{CH}$ смещается из точки $K$, вблизи которой формируется область отрицательных эффективных масс. В [5-9] эта особенность зонной структуры подтверждена экспериментально.

Исследования плазменных волн и коллективных возбуждений, называемых плазмонами, в 2D-дираковских кристаллах актуальны как с фундаментальной, так и с практической точек зрения. Фундаментальный интерес объясняется тем фактом, что такие квантово-механические объекты как плазмоны не имеют аналогов в классической теории. Более того, учет взаимодействия носителей заряда в 2D-дираковских кристаллах с плазмонами в ряде ситуаций является определяющим для объяснения особенностей плазменных колебаний [10], формирования электрон-плазмонных $[11,12]$ и магнитоплазмонных [13] комплексов. Практический интерес к таким объектам связан с возможностью увеличения быстродействия электронных устройств, использующих в своей работе плазмонную динамику $[14,15]$. Последнее объясняется тем фактом, что групповая скорость плазмонов превышает дрейфовую скорость электронов на несколько порядков.

Плазменные возбуждения в биграфене исследовались как в рамках гидродинамической модели [16], так и в приближении случайных фаз (ПСФ) [10,17-19]. В [20] проведен учет электрон-фононного взаимодействия. Плазменные колебания в биграфене без СН изучены в $[17,18,21]$ в рамках ПСФ. В [19] рассчитан закон дисперсии для плазмонов в биграфене с собственной проводимостью, когда уровень Ферми находится строго в точке $K$. В этой ситуации плазмоны образуются только 
при ненулевой температуре. Закон дисперсии плазмонов в биграфене с СН исследован в [10]. Однако в [10] предполагалось, что электронный газ является невырожденным (уровень Ферми лежал внутри запрещенной зоны). Кроме того, результаты [10] справедливы для малых значений $\mathrm{CH}: V_{0} \equiv e \varphi \ll \varepsilon_{\perp}$. Здесь $\varepsilon_{\perp} \sim 0.39 \mathrm{eV}-$ интеграл перекрытия волновых функций ближайших атомов из разных графеновых слоев, $\varphi=E d-\mathrm{CH}$, $E$ - напряженность поперечного электрического поля, $d \sim 0.34 \mathrm{~nm}$ - расстояние между графеновыми плоскостями. Ниже мы рассматриваем абсолютный ноль температуры как одну из возможных ситуаций, а также считаем, что уровень Ферми может попадать в зону проводимости биграфена. В такой ситуации электронный газ уже нельзя считать невырожденным, как это сделано в [10].

\section{1. Закон дисперсии для плазмонов в биграфене}

Считаем, что графеновые слои параллельны координатной плоскости $x y$. Квантово-механическое состояние электрона в биграфене вблизи точки $K$ описывается четырехкомпонентным спинором $\psi$, компоненты которого отвечают двум подрешеткам графена и двум его слоям [3]. Спинор $\psi$ удовлетворяет уравнению $i \hbar \partial_{t} \psi=\hat{H} \psi$, где гамильтониан $\hat{H}$ в приближении сильной связи имеет следующий вид

$$
\hat{H}=\left(\begin{array}{cccc}
\frac{V_{0}}{2} & v_{\mathrm{F}} \hat{\pi} & \varepsilon_{\perp} & 0 \\
\nu_{\mathrm{F}} \hat{\pi}^{+} & \frac{V_{0}}{2} & 0 & 0 \\
\varepsilon_{\perp} & 0 & -\frac{V_{0}}{2} & \nu_{\mathrm{F}} \hat{\pi}^{+} \\
0 & 0 & v_{\mathrm{F}} \hat{\pi} & -\frac{V_{0}}{2}
\end{array}\right),
$$

где $v_{\mathrm{F}}-$ скорость Ферми, $\hat{\pi}=\hat{p}_{x}+i \hat{p}_{y}$. Собственные значения гамильтониана (1) равны [3]

$$
\begin{gathered}
\varepsilon(\mathbf{p})= \pm \sqrt{\frac{\varepsilon_{\perp}^{2}}{2}+\frac{V_{0}^{2}}{4}+v_{\mathrm{F}}^{2} p^{2} \mp F(\mathbf{p})}, \\
F(\mathbf{p})=\sqrt{\frac{\varepsilon_{\perp}^{4}}{4}+\left(\varepsilon_{\perp}^{2}+V_{0}^{2}\right) v_{\mathrm{F}}^{2} p^{2}} .
\end{gathered}
$$

Так, например, согласно (2) энергия в точке $K$ для нижней дисперсионной ветви зоны проводимости равна $\varepsilon_{\mathrm{K}}=V_{0} / 2$. Далее полагаем, что электронный газ находится при абсолютном нуле температуры, а уровень Ферми лежит в зоне проводимости и не пересекает ее верхнюю дисперсионную ветвь. Это позволяет оставить в выражении (2) только верхние знаки.

В рамках ПСФ закон дисперсии для плазмонов находится из уравнения

$$
1-V(\mathbf{k}) \Pi(\omega, \mathbf{k})=0,
$$

где $V(\mathbf{k})=2 \pi e^{2} / k-$ потенциал неэкранированного взаимодействия электронов, которое предполагается куло-

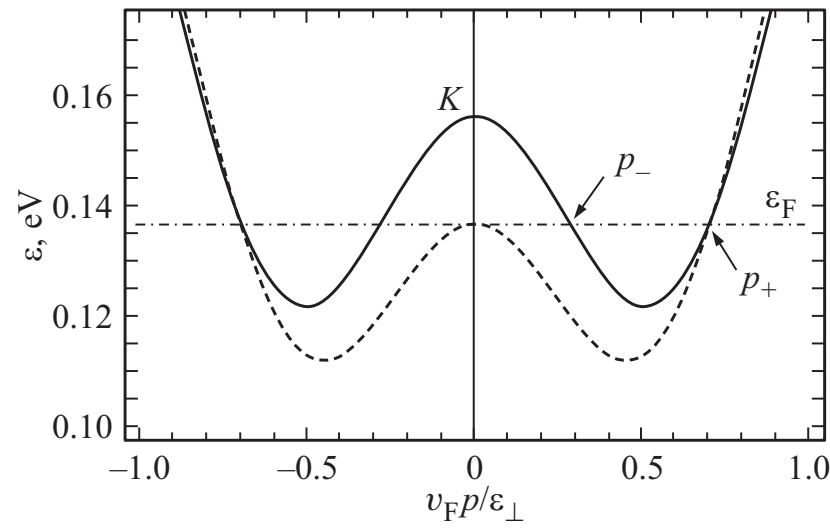

Рис. 1. Нижняя дисперсионная ветвь зоны проводимости биграфена с СН. Сплошная линия $-V_{0}=312 \mathrm{meV}$, пунктирная линия $-V_{0}=273 \mathrm{meV}$.

новским $[18,22]$, П $(\omega, \mathbf{k})$ - поляризационный оператор, вычисляемый по формуле

$$
\Pi(\omega, \mathbf{k})=i \int G\left(\varepsilon_{1}, \mathbf{p}_{1}\right) G\left(\varepsilon_{2}, \mathbf{p}_{2}\right) \frac{d \varepsilon d^{2} \mathbf{p}}{(2 \pi \hbar)^{3}},
$$

$G(\varepsilon, \mathbf{p})$ - причинная функция Грина для электрона, $\mathbf{p}_{1,2}=\mathbf{p} \pm \hbar \mathbf{k} / 2, \quad \varepsilon_{1,2}=\varepsilon \pm \hbar \omega / 2$. Считая температуры предельно низкими, получаем после некоторых преобразований следующее выражение:

$$
\begin{aligned}
\Pi(\omega, \mathbf{k})= & \frac{\theta\left(2 \varepsilon_{\mathrm{F}}-\Delta_{g}\right)}{2 \pi \hbar^{2}}\left[\frac{p_{+}}{\left|\nu_{+}\right|}\left(\frac{\omega}{\sqrt{\omega^{2}-v_{+}^{2} k^{2}}}-1\right)\right. \\
& \left.+\frac{p_{-}}{\left|v_{-}\right|}\left(\frac{\omega}{\sqrt{\omega^{2}-v_{-}^{2} k^{2}}}-1\right) \theta\left(V_{0}-2 \varepsilon_{\mathrm{F}}\right)\right] .
\end{aligned}
$$

Здесь $v=\partial_{p} \varepsilon(p), v_{ \pm}=v\left(p_{ \pm}\right), p_{ \pm}-$корни уравнения $\varepsilon(p)=\varepsilon_{\mathrm{F}}$ ( $p_{+}$лежит в области положительных эффективных масс, $p_{-}$лежит в области отрицательных эффективных масс, рис. 1), $\varepsilon(p)$ - электронный спектр (2), взятый с верхними знаками, $\varepsilon_{\mathrm{F}}-$ энергия Ферми, $\theta(x)$ - ступенчатая функция, $\Delta_{g}-$ энергетическая щель.

Известно, что в длинноволновом приближении закон дисперсии плазмонов в 2D-структурах имеет вид $\omega_{\mathbf{k}} \propto \sqrt{k}$, где $\mathbf{k}-$ волновой вектор плазмона. Коэффициент пропорциональности определяет групповую скорость плазменных волн и зависит от электронных свойств материала. Данный коэффициент является функцией $\mathrm{CH}$ и пропорционален кривизне дисперсионной линии плазмонов при $k=0$. Его вычисление является основной задачей в настоящей работе. Укажем на некоторые отличия решаемой здесь задачи от уже рассмотренных в предыдущих исследованиях. Во-первых, в отличие от [17-19,21] мы изучаем биграфен с $\mathrm{CH}$. Во-вторых, в случае предельно низких температур мы 
используем более общее выражение для электронного спектра, справедливое при $V_{0} \sim \varepsilon$, что позволяет в более широких пределах менять параметр $V_{0}$ по сравнению с [10]. В-третьих, в отличие от $[10,19]$ здесь считается, что уровень Ферми располагается в зоне проводимости.

В длинноволновом приближении вместо (5) приходим к следующему выражению:

$$
\Pi(\omega, \mathbf{k})=\frac{k^{2} \theta\left(2 \varepsilon_{\mathrm{F}}-\Delta_{g}\right)}{4 \pi \hbar^{2} \omega^{2}}\left(p_{+} \nu_{+}+p_{-}\left|\nu_{-}\right| \theta\left(V_{0}-2 \varepsilon_{\mathrm{F}}\right)\right) .
$$

С помощью соотношения (3) находим энергию плазмона

$$
\hbar \omega=e \theta\left(2 \varepsilon_{\mathrm{F}}-\Delta_{g}\right) \sqrt{\frac{k}{2}\left(p_{+} \nu_{+}+p_{-}\left|\nu_{-}\right| \theta\left(V_{0}-2 \varepsilon_{\mathrm{F}}\right)\right)} .
$$

Считаем далее, что уровень Ферми фиксирован и определяется концентрацией $n_{0}$ носителей заряда в зоне проводимости при $V_{0}=0$. Значения концентраций $n_{0}$ берутся по порядку величины равными тем, что использовались в недавних экспериментах [7,14]. Например, $n_{0}=2 \cdot 10^{12} \mathrm{~cm}^{-2}$, что соответствует $\varepsilon_{\mathrm{F}}=100 \mathrm{meV}$. Зависимости энергии плазмона от параметра $V_{0}$, построенные по формуле (7) для фиксированного значения волнового числа, показаны на рис. 2 и 3 сплошными линиями. Из рисунков видно, что энергия $\hbar \omega$, а вместе с тем и кривизна дисперсионной линии для плазмонов, меняются с изменением СН. Возможность такого эффекта предсказана в [10] для невырожденного электронного газа. Однако результаты, полученные в [10], справедливы для $V_{0} \ll \varepsilon_{\perp}$.

Из рис. 2 видна точка излома $A$ для функции $\hbar \omega\left(V_{0}\right)$, которая, согласно (7), достигается при $V_{0}=2 \varepsilon_{\mathrm{F}}$. Для наблюдения этого эффекта необходимо, чтобы при начальном (минимальном) значении $\mathrm{CH}$ уровень Ферми был выше значения $\varepsilon_{\mathrm{K}}$. В [10] уровень Ферми лежал внутри запрещенной зоны, а электронный газ полагался невырожденным, так что появление точки излома для функции $\hbar \omega\left(V_{0}\right)$ не могло быть предсказано в рамках теории [10]. Действительно, в [10] плазменная частота при фиксированном значении волнового числа монотонно убывала с ростом $\mathrm{CH}$.

\section{2. Влияние температуры на дисперсионную линию плазмонов}

Если температура близка к абсолютному нулю, то, как видно из графиков, представленных на рис. 2 и 3, при $\Delta_{g}>2 \varepsilon_{\mathrm{F}}$ происходит подавление плазменных колебаний. Действительно, в этом случае уровень Ферми оказывается внутри запрещенной зоны. Однако при ненулевых температурах концентрация электронов в зоне проводимости остается отличной от нуля, даже

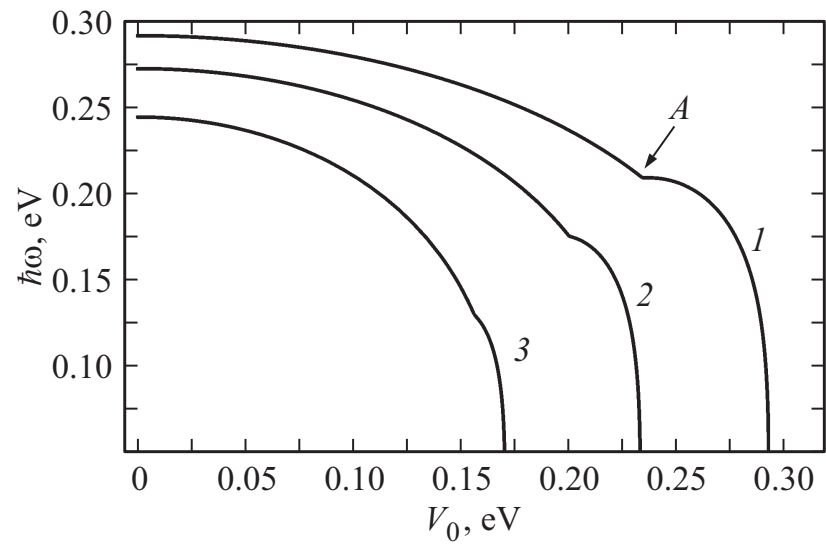

Рис. 2. Зависимость энергии плазмона от параметра $V_{0}$. $1-\varepsilon_{\mathrm{F}}=117 \mathrm{meV}, 2-\varepsilon_{\mathrm{F}}=100 \mathrm{meV}, 3-\varepsilon_{\mathrm{F}}=78 \mathrm{meV}$.

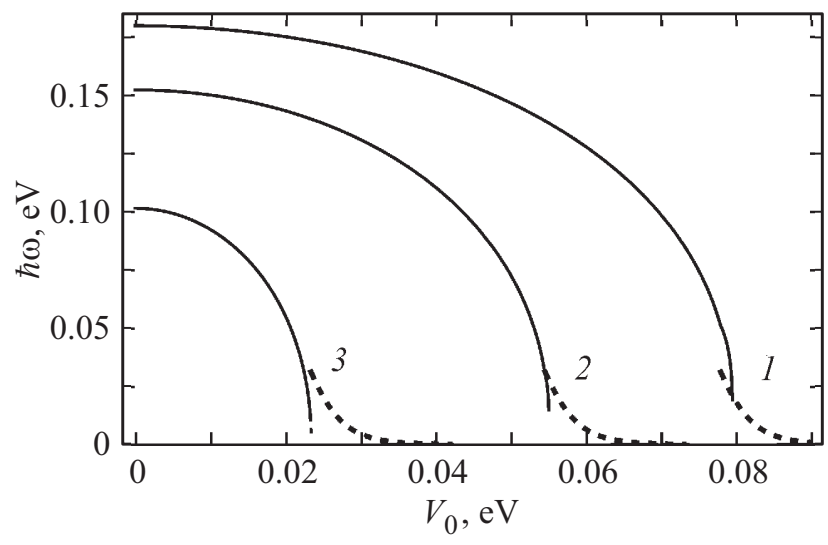

Рис. 3. Зависимость энергии плазмона от параметра $V_{0}$ (случай $\left.V_{0} \ll \varepsilon_{\perp}\right) .1-\varepsilon_{\mathrm{F}}=39 \mathrm{meV}, 2-\varepsilon_{\mathrm{F}}=27 \mathrm{meV}$, $3-\varepsilon_{\mathrm{F}}=12 \mathrm{meV}$. Пунктирные линии соответствуют температуре $10 \mathrm{~K}$.

если уровень Ферми располагается ниже дна этой зоны. Это приводит к размытию границы существования плазменных колебаний на графике зависимости $\hbar \omega\left(V_{0}\right)$. Для анализа температурной зависимости плазменной частоты, воспользуемся приближенным выражением для гамильтониана [23]:

$$
\hat{H}=\left(\begin{array}{cc}
\frac{V_{0}}{2} & \frac{v_{\mathrm{F}}^{2}\left(\hat{\pi}^{+}\right)^{2}}{\varepsilon_{\perp}} \\
\frac{v_{\mathrm{F}}^{2}(\hat{\pi})^{2}}{\varepsilon_{\perp}} & -\frac{V_{0}}{2}
\end{array}\right) .
$$

Несмотря на приближенную форму, гамильтониан (8) широко используется в аналитических расчетах в тех случаях, когда выполняется условие $V_{0} \ll \varepsilon_{\perp}$ (например, [24-26]). Собственные значения (8) равны

$$
\varepsilon=\frac{V_{0}}{2}\left(1+\frac{2 v_{\mathrm{F}}^{2} p^{4}}{\varepsilon_{\perp}^{2} V_{0}^{2}}\right)
$$


Считаем теперь, что система имеет конечную температуру. В длинноволновом приближении имеем

$$
\Pi(\omega, \mathbf{k})=-\frac{k^{2}}{4 \pi \hbar^{2} \omega^{2}} \int \frac{\partial n}{\partial \varepsilon} v(\varepsilon) p(\varepsilon) d \varepsilon
$$

Используя статистику Ферми-Дирака, получаем вместо (10)

$$
\Pi(\omega, \mathbf{k})=\frac{k^{2} T}{\pi \hbar^{2} \omega^{2}} \ln \left(1+e^{\frac{2 \varepsilon_{\mathrm{F}}-V_{0}}{2 T}}\right) .
$$

С помощью (3) находим энергию плазмона

$$
\hbar \omega=e \sqrt{2 T k \ln \left(1+e^{\frac{2 \varepsilon_{\mathrm{F}}-V_{0}}{2 T}}\right)} .
$$

Зависимости энергии плазмона от температуры вблизи границы подавления плазменных колебаний, построенные по формуле (12) для фиксированного значения волнового числа, показаны на рис. 3 пунктирными линиями.

\section{3. Заключение}

Выше исследовано влияние $\mathrm{CH}$ на дисперсионную линию для плазмонов в биграфене. При этом в противоположность [10] использовалось более общее выражение для электронного спектра биграфена, учитывающее в отличие от [17-19,20] поперечное электрическое поле и справедливое и для случая $V_{0} \sim \varepsilon_{\perp}$. Зависимость энергии плазмона $\hbar \omega$ от $\mathrm{CH}$ имеет немонотонный характер. Причиной появления точки излома $A$ (рис. 2) является наличие области отрицательных эффективных масс в окрестности точки $K$ (рис. 1). Действительно, энергетическая щель увеличивается с ростом $\mathrm{CH}$, что приводит к смещению вверх по энергии области отрицательных эффективных масс и приближению к уровню Ферми. Пока вся эта область остается ниже уровня Ферми, ее электроны не участвуют в формировании плазменных колебаний, и вклад в них дают только носители из области положительных эффективных масс (электроны с импульсом $p_{+}$). Как только уровень Ферми пересекает область отрицательных эффективных масс, в выражении (7) появляются дополнительные слагаемые, связанные с вкладом носителей заряда с импульсом $p_{-}$. Это приводит к возрастанию плазменной частоты при фиксированном значении волнового числа $k$. Вклад электронов с импульсами $p_{-}$становится возможным при конечном значении $\mathrm{CH} V_{0 A}$, соответствующем точке излома $A$ для функции $\hbar \omega\left(V_{0}\right)$.

Для наблюдения особенностей плазменных колебаний, описанных выше, уровень Ферми должен превышать энергию электронов в точке $K$ при начальном значении СН. Отметим, что в [10] электронный газ считался невырожденным, а уровень Ферми лежал внутри запрещенной зоны. Таким образом, появление точки излома для функции $\hbar \omega\left(V_{0}\right)$ не может быть предсказано в рамках теории [10].
В заключение отметим, что $\varepsilon_{\mathrm{F}}<\varepsilon_{\perp}$. Параметр $\varepsilon_{\perp}$ равен минимуму энергии для верхней дисперсионной ветви электронов в зоне проводимости, что оправдывает использование только верхних знаков в формуле (2).

\section{Финансирование работы}

Работа выполнена при финансовой поддержке Минобрнауки России в рамках проектной части государственного задания, код проекта: 3.2797.2017/4.6.

\section{Конфликт интересов}

Авторы заявляют, что у них нет конфликта интересов.

\section{Список литературы}

[1] M. Hofmann, W.-Y. Chiang, T.D. Nguyen, Y.-P. Hsieh. Nanotechnology 26, 335607 (2015).

[2] X. Guo, W. Wang, H. Nan, Y. Yu, J. Jiang, W. Zhao, J. Li, Z. Zafar, N. Xiang, Zhonghua Ni, W. Hu, Y. You, Zhenhua Ni. Optica 3, 1066 (2016).

[3] E. McCann, M. Koshino. Rep. Prog. Phys. 76, 056503 (2013).

[4] E.V. Castro, K.S. Novoselov, S.V. Morozov, N.M.R. Peres, J.M.B. Lopes dos Santos, J. Nilsson, F. Guinea, A.K. Geim, A.H. Castro Neto. J. Phys. Condens. Matter 22, 175503 (2010).

[5] T. Ohta, A. Bostwick, T. Seyller, K. Horn, E. Rotenberg. Science 313, 951 (2006).

[6] Z.Q. Li, E.A. Henriksen, Z. Jiang, Z. Hao, M.C. Martin, P. Kim, H.L. Stormer, D.N. Basov. Phys. Rev. Lett. 102, 037403 (2009).

[7] K.F. Mak, C.H. Lui, J. Shan, T.F. Heinz. Phys. Rev. Lett. 102, 256405 (2009).

[8] L.M. Malard, J. Nilsson, D.C. Elias, J.C. Brant, F. Plentz, E.S. Alves, A.H. Castro Neto, M.A. Pimenta. Phys. Rev. B 76, 201401(R) (2007).

[9] А.В. Елецкий, И.М. Искандарова, А.А. Книжник, Д.Н. Красиков. УФН 181, 233 (2011); [A.V. Eletskii, I.M. Iskandarova, A.A. Knizhnik, D.N. Krasikov. Phys. Usp. 54, 227 (2011).].

[10] П.В. Бадикова, С.Ю. Глазов. Изв. РАН. Сер. физ. 81, 59 (2017); [P.V. Badikova, S.Yu. Glazov. Bull. Russ. Acad. Sci.: Phys. 81, 51 (2017).].

[11] A. Bostwick, F. Speck, T. Seyller, K. Horn, M. Polini, R. Asgari, A.H. MacDonald, E. Rotenberg. Science 328, 999 (2010).

[12] P.K. Pyatkovskiy, T. Chakraborty. Phys. Rev. B 93, 085145 (2016).

[13] Y.E. Lozovik, A.A. Sokolik. Nanoscale Res. Lett. 7, 134 (2012).

[14] A.N. Grigorenko, M. Polini, K.S. Novoselov. Nature Photonics 6, 749 (2012).

[15] F. Peragut, L. Cerutti, A. Baranov, J.P. Hugonin, T. Taliercio, Y. De Wilde, J.J. Greet. Optica 4, 1409 (2017).

[16] N.M. Hassan, V.V. Mkhitaryan, E.G. Mishchenko. Phys. Rev. B 85, 125411 (2012). 
[17] G. Borghi, M. Polini, R. Asgari, A.H. MacDonald. Phys. Rev. B 80, 241402(R) (2009).

[18] R. Sensarma, E.H. Hwang, S. Das Sarma. Phys. Rev. B 82, 195428 (2010).

[19] S. Das Sarma, Q. Li. Phys. Rev. B 87, 235418 (2013).

[20] T. Low, F. Guinea, H. Yan, F. Xia, P. Avouris. Phys. Rev. Lett. 112, 116801 (2014).

[21] P.M. Krstajic, F.M. Peeters. Phys. Rev. B 88, 165420 (2013).

[22] S. Das Sarma, E.H. Hwang. Phys. Rev. Lett. 102, 206412 (2009).

[23] A.V. Rozhkov, A.O. Sboychakov, A.L. Rakhmanov, F. Nori. Phys. Rep. 648, 1 (2009).

[24] M. Barbier, P. Vasilopoulos, F.M. Peeters. Phys. Rev. B 82, 235408 (2010).

[25] M. Killi, S. Wu, A. Paramekanti. Phys. Rev. Lett. 107, 086801 (2011).

[26] F. Sattari, E. Faizabadi. Int. J. Mod. Phys. B 27, 1350024 (2013).

Редактор Е.Ю. Флегонтова 